\title{
Programa de tutoria por pares no ensino superior: Estudo de caso
}

\author{
Carolina Estevam \\ Ana Júlia Basilio \\ Marina Greghi Sticcal \\ Fabiana Maris Versuti \\ Universidade de São Paulo, Ribeirão Preto-SP, Brasil
}

\section{Resumo}

A literatura aponta que o período de transição entre o ensino médio e o ensino superior tem gerado dificuldades de adaptação dos universitários. Os programas de tutoria por pares vêm ganhando espaço em diversos países devido aos bons resultados constatados após a sua aplicação no bem-estar e processo de adaptação dos universitários recémingressos. O presente estudo tem como objetivo apresentar um programa de tutoria por pares no ensino superior. Participaram deste estudo 18 estudantes universitários, sendo que 9 exerceram o papel de tutor e 9 de tutorando. Os participantes avaliaram positivamente o programa, para os tutores atendeu suas demandas iniciais ao fornecer informações em relação à universidade e ao curso, gestão de tempo e métodos de estudo, para os tutores possibilitou o contato com a prática.

Palavras-chave: Tutoria entre pares, ensino superior, estudantes universitários, programas educacionais

\section{Abstract: Peer tutoring program in higher education: A case study}

The literature shows that the transition period between high school and higher education has generated difficulties in adapting university students. Peer tutoring programs have been gaining ground in several countries due to the good results found to the well-being and adaptation process of newcomers. The present study aims to present a peer tutoring program in higher education. A total of 18 college students participated in this study, 9 of which were tutor and 9 tutoring students. Participants positively evaluated the program, for tutors met their initial demands by providing information regarding university and course, time management and study methods, to the tutors made it possible to contact with the practice.

Keywords: Peer tutoring, high education, college students, educational programs

\section{Resumen: Programa de tutoría por pares en la enseñanza superior: Estudio de caso}

La literatura apunta que el período de transición entre la enseñanza media y la enseñanza superior ha generado dificultades de adaptación de los universitarios. Los programas de tutoría por pares vienen ganando espacio en diversos países debido a los buenos resultados constatados después de su aplicación en el bienestar y proceso de adaptación de los universitarios recién ingresados. El presente estudio tiene como objetivo presentar un programa de tutoría por pares en la enseñanza superior. Participaron de este estudio 18 estudiantes universitarios, siendo que 9 ejercieron el papel de tutor y 9 de tutorando. Los participantes evaluaron positivamente el programa, para los tutores atendió sus demandas iniciales al proporcionar informaciones en relación a la universidad y al curso, gestión de tiempo y métodos de estudio, para los tutores posibilitó el contacto con la práctica.

Palabras claves: Tutoría por pares, enseñanza superior, estudiantes universitarios, programas educacionales

\footnotetext{
${ }^{1}$ Endereço para correspondência: Universidade de São Paulo. Faculdade de Filosofia, Ciências e Letras de Ribeirão Preto. Departamento de Psicologia e Educação. Avenida Bandeirantes, 3900, 14040-901, Ribeirão Preto, SP. Fone: 163915 9095. E-mail: marinagreghi@ffclrp.usp.br
} 
No Brasil, há pouco menos de uma década houve um aumento significativo do número de alunos ingressando no ensino superior, expansão dos cursos universitários em termos de estrutura física, recursos humanos, criação de novos cursos e aumento de vagas ofertadas em universidades públicas e privadas do país. Com essa ampliação, iniciou-se o processo de democratização do acesso à universidade, contribuindo para a diversidade de perfis de alunos nesse contexto (Oliveira \& Morais, 2015). Contudo, outro fenômeno observado refere-se a um crescimento da taxa de insucesso e desistência escolar, que vem aumentando progressivamente não apenas no Brasil, mas também em outros países mais desenvolvidos (Gorlitz \& Gravert, 2016).

De acordo com Meneses (2018), a taxa de desistência acumulada em cinco anos no ensino superior brasileiro para os que entraram na faculdade em 2010 foi de $49 \%$. No setor privado a evasão foi de $53 \%$, ao passo que nas faculdades públicas a desistência foi de $40 \%$. Segundo revisão da literatura, as dificuldades na transição do Ensino Médio para a Universidade (Almeida, Ferreira, \& Soares, 1999) e na adaptação à instituição ou no relacionamento com a comunidade acadêmica em geral são fatores que impactam na construção do fenômeno da evasão no ensino superior (Diniz \& Almeida, 2006; Mercuri \& Polydoro, 2003).

A transição dos jovens para o ensino superior suscita, por um lado, inúmeras expectativas positivas e, por outro, receios e dificuldades (Almeida, Soares, \& Ferreira, 2002). A adaptação ao ambiente universitário promove diversas mudanças da vida dos sujeitos, uma parte implica a acomodação de novos hábitos, e outra requer a incorporação e desenvolvimento de novos conhecimentos e habilidades (Oliveira \& Dias, 2014).

Almeida et al. (1999) ao discorrerem sobre o período de escolarização dos sujeitos consideram diversas variáveis que interagem na vida do aluno favorecendo ou dificultando a adaptação dos mesmos e as classificam em cinco dimensões, sendo elas: pessoal, interpessoal, estudo, carreira e institucional. A dimensão pessoal envolve aspectos que abrangem tanto o bem estar físico quanto psicológico do estudante, como hábitos de sono, saúde, autonomia, alimentação, autoconfiança, equilíbrio emocional, entre outros. A dimensão interpessoal reúne variáveis alusivas ao relacionamento entre colegas, sejam situações de cooperação ou amizades mais significativas. Além disso, inclui o envolvimento com atividades extracurriculares, como pertença a entidades, participação de atividades culturais e recreativas, por exemplo. A dimensão estudo compreende questões de ordem acadêmica, tais quais, competência de estudo e a capacidade de organizar-se enquanto estudante. A dimensão carreira engloba fatores como expectativas, aprendizagens, competências e satisfação, contribuindo para o desenvolvimento da identidade e adaptação do estudante. Por fim, a dimensão institucional contempla pontos que se associam ao conhecimento dos serviços e infraestrutura da instituição. Em outras palavras, conhecimento dos serviços e infraestrutura da instituição são aspectos de grande influência no sentimento de querer pertencer-se àquele determinado local ou não (Almeida et al., 1999).

Um estudo desenvolvido por Oliveira e Morais (2015), em uma universidade federal do Estado do Paraná, observou que os participantes obtiveram menores índices de adaptação nas dimensões pessoal e estudo, o que expressa dificuldades na gestão do tempo, disposição, organização pessoal, autonomia, ou seja, fatores próprios de cada sujeito. Outro estudo, todavia, apontou que estudantes que obtiveram índices mais elevados nas mesmas dimensões apresentaram um rendimento acadêmico superior aos demais (Cunha \& Carrilho, 2005).

Em suma, o ingresso na universidade pode ser uma condição bastante estressora para os jovens universitários. Assim sendo, estudos focados na compreensão deste processo, nas dificuldades e nos impactos dessa experiência nas esferas psicológica e social, mostram-se pertinentes e urgentes (Teixeira, Dias, Wottrich, \& Oliveira, 2008).

Vale destacar, que não basta inserir o aluno na universidade, são imprescindíveis intervenções tanto de cunho político-social quanto psicopedagógico, a fim de minimizar o grande número de evasão dos ingressantes, bem como os impactos das incidências de baixo rendimento acadêmico (Oliveira \& Morais, 2015). Neste sentido, Millan, Souza, De Marco, Rossi e Arruda (1998) apontam que desde a década de 50 existe uma preocupação em algumas universidades brasileiras em desenvolver ações voltadas para a assistência psicológica ao estudante universitário e destaca intervenções realizadas, tais como o Grupo de Assistência Psicológica ao Aluno da USP-SP e o Serviço de Atendimento Psicológico e Psiquiátrico ao Estudante da UNICAMP.

No âmbito da assistência aos universitários, uma ação que merece destaque é o "programa de tutoria por pares", o qual vem ganhando crescente espaço em diversos países (Eby, Rhodes, \& Allen, 2007). Tal modelo é tradicionalmente reconhecido em universidades da Austrália, Canadá, Estados Unidos e Inglaterra, além de universidades portuguesas (Faria, Coelho, Oliveira, Lucas, \& Soares, 2014), italianas (Pedicchiio \& Fontana, 2000; Munley, Garvey, \& McConnell, 2010), belgas (De Smet, Van Keer, \& Valcke, 2009), dinamarquesas (Lueg \& Lueg, 2014), norueguesas (Qureshi, \& Stormyhr, 
2012), do Sul da África (Underhill \& McDonald, 2010) e universidades da América Latina, como uruguaias (Collazo, Hernández, \& Seoane, 2014) e colombianas (Cardozo-Ortiz, 2011). Contudo no Brasil, os estudos ainda são incipientes.

A revisão da literatura aponta que os programas de tutoria por pares apresentam diferentes estruturas, entretanto, pode-se observar alguns aspectos em comum entre todos. Dos aspectos comuns destacam-se: a definição de papéis sociais e relacionais na busca do objetivo em comum, que consiste na proteção e adaptação do tutorando; os tutores apresentam maior experiência e influência na realização de algo em um ambiente específico; o benefício na tutoria é mútuo para ambas as partes (tutor e tutorando) (Jacobi, 1991; Topping, 1996; Wilson \& Arendale, 2011; Topping, Miller, Thurston, McGavock, \& Conlin, 2011).

Em um estudo realizado na Universidade de Quebec foram identificados diversos benefícios trazidos pela utilização de um programa de tutoria na universidade. Os alunos tutorandos apresentaram, após a participação no programa, índices mais altos de motivação, como também maior engajamento institucional e adaptação social (Larose et al., 2011). Outro estudo realizado na Universidade Northern Kentucky, nos Estados Unidos, mostra a influência positiva de um programa de tutoria por pares na diminuição da taxa de evasão escolar de alunos recém-ingressos (Bowling, Doyle, Taylor, \& Antes, 2015). Além disso, a pesquisa realizada no Imperial College London aponta para a eficiência de programas de tutoria enquanto instrumentos de alto desempenho para o esquema ensino-aprendizagem (Alpay, Cutler, Eisenbach, \& Field, 2010), demonstrando a variedade de resultados que a implementação de um programa de tutoria pode trazer.

O programa de tutoria descrito nesta investigação adotou o modelo em que aluno de nível mais avançado no curso (veterano) ocupa o papel de tutor, o qual fornece suporte a um aluno recém-ingresso (calouro). Segundo Muñoz, Barbero e Feliu (2015), tal proposta pode ajudar as universidades a diminuir a taxa de evasão dos novos estudantes, bem como contribuir para o sucesso adaptativo destes. Além disso, o mesmo estudo aponta que não apenas a universidade e o tutorando se beneficiam com o programa. O próprio tutor obtém ganhos, considerando $\mathrm{o}$ aprofundamento de conhecimento que o aluno adquire nos temas trabalhados e o desenvolvimento de habilidades sociais (Garitano, Ruiz, \& Andonegui, 2017).

Considerando os benefícios do modelo de tutoria por pares para o processo de adaptação à vida universitária apresentados pela literatura relacionados em especial com: 1. Desenvolvimento de habilidades acadêmicas e sociais; 2 . Clima e na disciplina na sala de aula; 3. Aprimoramento das relações entre pares; 4. Facilitação do processo de transição do ensino médio para o ensino superior; 5. Potencialização de vínculos do aluno com o ambiente universitário; 6. Desempenho acadêmico e; 7. Autoestima dos alunos (Muñoz, Barbero, \& Feliu, 2015). O modelo foco desta investigação almejou contemplar tais benefícios, neste sentido, o objetivo principal deste estudo é descrever um programa de tutoria por pares no ensino superior brasileiro de um curso de graduação em Psicologia.

\section{Método}

\section{Participantes}

Participaram deste estudo piloto 18 estudantes universitários de um curso de Psicologia de uma universidade pública, sendo que 9 alunos exerceram o papel de tutor e 9 o papel de tutorando. Dos 9 tutorandos participantes, 7 eram do sexo feminino e 2 do sexo masculino. Observou-se idade mínima de 18 e máxima de 21 anos entre os tutorandos, além de mais da metade $(57,1 \%)$ ter cursado outra faculdade previamente, mas não a concluíram, e frequentado curso pré-vestibular. Quanto aos tutores, também mais da metade frequentou o curso pré-vestibular $(57,1 \%)$, porém apenas uma parcela $(28,6 \%)$ cursou outra faculdade previamente sem concluí-la. A idade mínima é de 18 anos e máxima de 30 anos. Havia um aluno do sexo masculino participando enquanto tutor e 8 se declararam do sexo feminino.

\section{Instrumentos}

Inicialmente, foi realizada uma entrevista para identificar as principais demandas dos alunos em relação ao processo de adaptação à vida universitária. Para realizar a avaliação pré-intervenção e pós-intervenção, utilizou-se os seguintes instrumentos:

Inventário de Habilidades Sociais: IHS-Del-Prette, desenvolvido por Del Prette e Del Prette (2001). O IHS-Del Prette é um instrumento constituído por 38 itens que têm como objetivo avaliar dimensões situacionais e comportamentais das habilidades sociais. Os itens são agrupados em cinco fatores denominados de F1 - enfrentamento e autoafirmação com risco, F2 - autoafirmação na expressão de sentimento positivo, F3 - conversação e desenvoltura social, F4 - autoexposição a desconhecidos e situações novas e F5 - autocontrole da agressividade. Os respondentes devem indicar a frequência com que agem ou sentem que recebem as classificações, de acordo com a indicação: A (nunca ou raramente), B (com pouca frequência), 
C (com regular frequência), D (muito frequentemente), E (sempre ou quase sempre). Conforme Bandeira, Neves, Pereira, Del Prette e Gerk (2000), o IHS-Del Prette possui validade concomitante com a Escala de Assertividade Rathus $(r=0,81, p=0,01)$.

Questionário de Vivências Acadêmicas: o Questionário de Vivências Acadêmicas (QVA) desenvolvido por Almeida, Ferreira e Soares (1999) é originalmente composto por 170 itens. No entanto, utilizou-se o questionário em uma versão reduzida, avaliando áreas como relacionamento com a família, professores e colegas, autonomia pessoal, autoconfiança, métodos de estudo, adaptação ao curso e à instituição, bem como bem-estar psicológico e gestão de tempo. As questões foram definidas visando adaptar ao contexto da própria universidade em que se instituiu o programa de tutoria. A escala de avaliação foi do tipo Likert de concordância e mensura o grau com que um atributo está presente no comportamento dos estudantes, segundo suas próprias percepções. Na escala, os respondentes classificaram os indicadores de cada categoria com o nível de concordância, sendo 1 (concordo totalmente) e 5 (discordo totalmente), para cada um dos itens indicados.

Roteiro de Entrevista Semiestruturado (Tabela1): foi elaborado um roteiro de entrevista semiestruturado com base nas dificuldades de adaptação encontradas em revisão da literatura. O roteiro era composto de questões relacionadas a: (a) expectativas em relação ao programa de tutoria por pares; (b) diferenças entre ensino médio e superior; c) avaliação em relação ao curso; (d) universidade; (e) relacionamento com colegas e docentes; (f) aspectos familiares; (g) questões pessoais e (h) habilidades sociais.

Tabela 1

Roteiro de Entrevista

\begin{tabular}{|c|c|}
\hline Dimensões & Questões \\
\hline Expectativas em relação ao programa & Quais são suas expectativas em relação ao programa? \\
\hline Diferenças entre ensino médio e ensino superior & $\begin{array}{l}\text { Quais foram as principais diferenças entre } \\
\text { o ensino médio e o ensino superior? }\end{array}$ \\
\hline Avaliação em relação ao curso & $\begin{array}{l}\text { Como está sendo seu primeiro ano no curso de psicologia? } \\
\text { Você conhece como funciona seu curso? } \\
\text { Quais as principais dúvidas? }\end{array}$ \\
\hline Universidade & $\begin{array}{l}\text { Quais as maiores dificuldades ao entrar na universidade? } \\
\text { Como foi o acolhimento da universidade? }\end{array}$ \\
\hline Relacionamento com colegas e docentes & $\begin{array}{l}\text { Como é seu relacionamento com seus colegas de turma? } \\
\text { Qual a sua percepção em relação aos docentes do curso? }\end{array}$ \\
\hline Aspectos familiares & $\begin{array}{l}\text { A entrada na universidade alterou a relação com seus pais? } \\
\text { Sem sim, de que forma? } \\
\text { Qual a opinião da sua família em relação a sua escolha profissional? }\end{array}$ \\
\hline Questões pessoais e habilidades sociais & $\begin{array}{l}\text { Como tem sido conciliar a faculdade com a vida pessoal? } \\
\text { Quais características individuais você considera que tem } \\
\text { ajudado na adaptação e quais tem causado dificuldades? }\end{array}$ \\
\hline
\end{tabular}

\section{Procedimentos}

Os estudantes universitários que assumiram o papel de tutorandos foram convidados a participar da intervenção por meio de divulgação em sala de aula e cartazes colocados no departamento de Psicologia. A intervenção foi conduzida por alunos do terceiro ano que foram matriculados na disciplina-estágio intitulada
Gestão de Carreira II, que contempla uma parte teórica e outra prática. Um dos pré-requisitos para ser tutor no programa era ter cursado a disciplina teórica intitulada Gestão de Carreira I que tinha como objetivo discutir questões relativas ao desenvolvimento da carreira, adaptação à vida universitária e identidade profissional do psicólogo, bem como determinar objetivos futuros para a sua vida acadêmica e profissional. Além da parte teórica, 
que consistiu no estudo de artigos sobre programas de tutoria por pares e adaptação à vida universitária, bem como análise dos principais instrumentos adotados como medida pré e pós intervenção, e no desenvolvimento dos materiais instrucionais que compuseram os quatro módulos do programa e norteavam as interações entre os tutores e tutorandos, tais como, textos e vídeos, a parte prática contemplava supervisões coletivas semanais e desenvolvimento das sessões de tutoria. Os tutores/estagiários entregavam antes de cada encontro um relatório da sessão realizada e com base neste material eram discutidas em grupo: as demandas de cada tutorando, o conteúdo da sessão, bem como possíveis dificuldades enfrentadas e possibilidades de manobras quanto à condução da próxima sessão.

A intervenção foi realizada individualmente por um único tutor ao longo de seis sessões, com periodicidade semanal e com duração média de sessenta minutos, sendo duas delas presenciais - de avaliação e fechamento - e as outras quatro foram sessões virtuais, realizadas por meio da utilização de redes sociais Facebook, Whatsapp e Hangouts. A escolha desta estrutura do programa foi feita com base na revisão de estudos internacionais e nacionais que apresentavam benefícios de encontros individualizados, com duração curta e peridiocidade semanal. Quanto à opção por sessões virtuais, foi um recurso sugerido pelos próprios tutorandos e tutores no inicio do programa. Era oferecida a opção de realizar encontros presenciais para os tutorandos que tivessem interesse neste formato.

No primeiro encontro foi feita a avaliação inicial dos participantes em relação aos problemas de adaptação à vida universitária e a entrevista com base no Roteiro semiestruturado. Além disso, foi aplicado o Questionário de Vivências Acadêmicas e o Inventário de Habilidades Sociais.

Em cada encontro, um tema relativo à adaptação à vida universitária foi abordado. Os temas foram definidos de acordo com as demandas principais identificadas na entrevista inicial e na revisão da literatura que apontavam as principais dimensões envolvidas na adaptação ao ensino superior de estudantes brasileiros e estruturado em módulos. Ao final de cada módulo os tutores redigiram um relatório a respeito da atividade realizada, cujo conteúdo abordou os principais pontos de cada sessão e as impressões do tutorando sobre o módulo. Os relatórios foram compartilhados com todos os estagiários antes das sessões de supervisão.

A sequência dos temas abordados no programa de tutoria por pares e os objetivos de cada módulo podem ser identificados na Tabela 2.

Tabela 2

Proposta de intervenção

\begin{tabular}{ll}
\hline \multicolumn{1}{c}{ Encontros Semanais } & \multicolumn{1}{c}{ Objetivos dos encontros } \\
\hline Encontro 1 (presencial) & $\begin{array}{l}\text { Identificar as demandas iniciais dos tutorandos, aplicar instrumentos, apresentar } \\
\text { os objetivos e funcionamento de um programa de tutoria por pares. }\end{array}$ \\
Encontro 2 (virtual) - Módulo 1 & $\begin{array}{l}\text { Apresentar aspectos do ambiente acadêmico, com descrições das oportunidades } \\
\text { e serviços oferecidos pelo curso de Psicologia da universidade favorecendo a } \\
\text { adaptação à dimensão institucional. }\end{array}$ \\
Encontro 3 (virtual) - Módulo 2 & $\begin{array}{l}\text { Apresentar conceitos relativos às dimensões de adaptação (pessoal, interpessoal, } \\
\text { carreira, estudo e institucional) e avaliar como o tutorando percebe sua } \\
\text { adaptação a tais dimensões, além de introduzir a importância da construção de } \\
\text { uma rede de apoio. }\end{array}$
\end{tabular}

Encontro 4 (virtual) HS - Módulo 3 Apresentar comportamentos envolvidos nas Habilidades Sociais e relação com o desempenho acadêmico e profissional. Trabalhar questões relacionadas ao planejamento e organização dos estudos.

Encontro 5 (virtual) - Módulo 4

Apresentar conceitos relacionados a Habilidades Sociais, tais como autorregulação, autoeficácia, assertividade, passividade e agressividade.

Encontro 6 (presencial)

Reaplicar instrumentos, realizar uma entrevista final com o objetivo de avaliar a satisfação em relação ao programa e identificar se os objetivos foram alcançados. 


\section{Tratamento e Análise dos Dados}

Os dados quantitativos foram tratados e analisados apenas por meio da estatística descritiva. Esta análise inicial consistiu em descrever as características gerais da amostra, no que tange à frequência de dados categoriais ou ordinais. Especificamente para o IHS-Del Prette, houve a organização por fatores e identificaram-se as classificações previstas no instrumento.

Os dados qualitativos foram analisados segundo pressupostos da análise do discurso do sujeito coletivo proposta por Lefèvre, Lefèvre e Teixeira (2000).

\section{Considerações Éticas}

No que diz respeito aos aspectos éticos, o projeto foi aprovado pelo Comitê de Ética em Pesquisa da Faculdade de Filosofia, Ciências e Letras de Ribeirão Preto - USP e previamente à aplicação dos questionários os participantes foram esclarecidos sobre os objetivos da pesquisa e seus procedimentos. O Termo de Consentimento Livre e Esclarecido foi distribuído para todos os participantes. Foi assegurada aos tutorandos e aos tutores a manutenção de sigilo e anonimato acerca de sua identidade, o caráter de sua participação voluntária e solicitada a autorização do uso de suas informações no estudo.

\section{Resultados}

\section{Demandas dos tutorandos e Condução das Sessões}

Conforme descrito nos procedimentos do estudo, foi realizado um levantamento de informações, com objetivo de identificar as principais demandas em relação à vida universitária e às habilidades sociais dos tutorandos. Os dados da avaliação inicial mensuram as respostas de apenas 6 tutorandos, os quais aceitaram identificar-se no QVA. Essa análise revelou que as dimensões que foram pontuadas com maior frequência por apresentarem dificuldades na adaptação foram: autonomia pessoal $(M=2.75)$, métodos de estudo $(M=2.83)$, adaptação à instituição $(M=2.16)$ e ao curso $(M=2.58)$, gestão de tempo $(M=2.94)$ e autoconfiança $(M=1.66)$.

A partir das entrevistas iniciais verificou-se que as principais demandas estavam relacionadas à falta de informação em relação à universidade e ao próprio curso, e demandas relacionadas a habilidades sociais, conforme especificado na Tabela 3. Tais dados estão de acordo com as demandas identificadas a partir do Questionário de Vivências Acadêmicas.

Tabela 3

Categorização das demandas iniciais dos tutorandos

\begin{tabular}{lccc}
\hline \multicolumn{1}{c}{ Tipo de demanda } & Frequência & $\begin{array}{c}\% \text { da } \\
\text { amostra total }\end{array}$ & \multicolumn{1}{c}{ Falas Ilustrativas } \\
\hline $\begin{array}{l}\text { Falta de informação em } \\
\text { relação à universidade }\end{array}$ & 2 & $8,7 \%$ & $\begin{array}{l}\text { "Principalmente, para conhecer mais a faculdade. Eu } \\
\text { entrei na quarta chamada e não participei da semana } \\
\text { de recepção dos calouros. Então, eu sou perdida com } \\
\text { relação aos projetos, entidades da faculdade." }\end{array}$
\end{tabular}

Falta de informação

em relação ao curso

Problemas com a didática dos professores

Dificuldade em fazer amizades
3

$13,0 \%$

4

$17,4 \%$

$2 \quad 8,7 \%$
"A principal queixa é sobre a falta de informação, é como se os professores e a própria faculdade agissem como se todos soubessem, e que obrigação é apenas do aluno em se informar e ir atrás, eu não sei nada sobre iniciação científica, bolsa e outras informações."

\section{"Gostaria que o programa me ajudasse a entender como vai ser o curso, pois tenho encontrado bastante dificuldade em relação aos professores, principalmente no que tange aos seus métodos utilizados e o que eles esperam de um aluno de graduação."}

"Entrevistador: Ah, então vocêfez amigos? Sim, alguns. Mas o pessoal da minha sala é muito unido, então se a gente vai para uma festa e se encontra, fica todo mundo junto. É que eu sou muito fechada, então não faço amizade muito facilmente, então eu diria que esse seria um problema na faculdade que eu já esperava." 
Tabela 3 (continuação)

Categorização das demandas iniciais dos tutorandos

\begin{tabular}{|c|c|c|c|}
\hline Tipo de demanda & Frequência & $\begin{array}{c}\% \text { da } \\
\text { amostra total }\end{array}$ & Falas Ilustrativas \\
\hline $\begin{array}{l}\text { Declarar ter duas vidas: uma } \\
\text { na faculdade e outra na casa natal }\end{array}$ & 2 & $8,7 \%$ & \\
\hline Dificuldades de ser assertivo & 4 & $17,4 \%$ & \\
\hline \multirow[t]{2}{*}{ Gestão do tempo } & 6 & $26,0 \%$ & $\begin{array}{l}\text { "Está sendo mais puxado do que eu esperava, não } \\
\text { dá tempo de fazer muitas coisas nem de ler todos os } \\
\text { textos, mas muita gente fala que o primeiro ano de } \\
\text { faculdade é mais chatinho e muito teórico, então eu } \\
\text { estou com expectativa para os próximos anos." }\end{array}$ \\
\hline & & & $\begin{array}{l}\text { "O que me pega é assim, a gente fica o dia inteiro } \\
\text { aqui na faculdade, a gente sai as } 6 \text { da tarde e assim, } \\
\text { as atividades não se encerram aqui, a gente tem que } \\
\text { chegar em casa e fazer muita coisa ainda, então eu } \\
\text { acho bem ruim, porque, por exemplo, eu estou agora } \\
\text { assim, mas nesse fim de semestre eu estou em aula, } \\
\text { mas estou fazendo coisa de outra matéria, ou eu falto } \\
\text { na aula pra estudar sabe? Coisas que eu acho que (?) } \\
\text { e assim, eu me sinto até mal, porque às vezes eu estou } \\
\text { na aula e falo "poxa, até queria prestar atenção, mas } \\
\text { eu preciso entregar isso amanhã", sabe?" }\end{array}$ \\
\hline
\end{tabular}

Quanto às classificações previstas no Inventário de Habilidades Sociais, dos 9 universitários participantes deste estudo, 2 receberam indicação final para Treinamento em Habilidades Sociais. Os dados da avaliação dos alunos a partir da aplicação do IHS podem ser vistos na Tabela 4.

A partir das entrevistas iniciais, as principais demandas dos participantes em relação às habilidades sociais e a frequência em que cada uma foi citada no relato dos participantes foram verificadas: dificuldade em iniciar, manter e encerrar uma conversação (1), baixa auto-eficácia (2); agressividade (1), dificuldades em ser assertivo (1) e dificuldade em lidar com a raiva (1). A partir dos dados levantados na primeira sessão e da revisão da literatura foram definidos os temas e objetivos de cada encontro e módulo.

No segundo encontro correspondente ao módulo 1, intitulado Vida Universitária, foram apresentadas informações gerais a respeito da Universidade, como também conhecimentos específicos sobre a Faculdade e o curso. Foi elaborado pelos alunos um Manual com informações sobre a universidade, seus serviços, sobre o curso e seu funcionamento, que foi disponibilizado antes do encontro. O material tinha o objetivo de funcionar como disparador temático para iniciar a interação do tutor e tutorando, sendo que o tutor iniciava o encontro enfocando em aspectos que interessavam o aluno e/ou que os tutorandos tinham dúvidas a respeito.

No terceiro encontro correspondente ao módulo 2, Adaptação à Universidade, o conteúdo abrangeu as dimensões de adaptação (pessoal, interpessoal, carreira, estudo e institucional) e a avaliação do próprio tutorando dessas dimensões na sua adaptação à universidade. Também foram elaborados vídeos com relatos de estudantes contando a respeito das dificuldades enfrentadas dentro de cada dimensão da adaptação e proposta uma atividade intitulada "mapa em rede", que visava identificar a rede de apoio dos estudantes.

Nos encontros 4 e 5 , correspondentes ao módulo 3 - Conhecimento de si mesmo, foram apresentadas questões sobre o conceito de habilidades sociais e sua relação com o desempenho profissional e o módulo 4 - Organização dos estudos, abordou os temas autorregulação, planejamento e organização, sendo esses relacionados diretamente ao desempenho acadêmico. Também foram disponibilizadas tabelas para a organização de estudo, possibilitando que os tutorandos iniciassem a cultura de planejamento.

$\mathrm{Na}$ última sessão, foi realizada uma devolutiva em relação ao IHS aplicado na primeira sessão, sendo que a partir desta devolutiva eram trabalhados os conteúdos com maior demanda dos participantes em relação às Habilidades Sociais. 
Tabela 4

Resultados do Inventário de Habilidades Sociais

\begin{tabular}{lcc}
\hline \multirow{2}{*}{ Fatores } & Avaliação Inicial & Frequência \\
\cline { 2 - 3 } & Classificação do repertório & 3 \\
F1: Enfrentamento e autoafirmação com risco & Elaborado & 5 \\
F2: Autoafirmação na expressão de sentimento positivo & Bom & 1 \\
& Baixo & 3 \\
F3: Conversação e desenvoltura social & Elaborado & 5 \\
& Bom & 1 \\
F4: Autoexposição a desconhecidos e situações novas & Baixo & 4 \\
& Elaborado & 1 \\
F5: Autocontrole da agressividade & Bom & 4 \\
& Baixo & 2 \\
Indicação Final & Elaborado & 4 \\
& Bom & 3
\end{tabular}

\section{Avaliação da satisfação do Programa de Tutoria por Pares para os Tutorandos}

Conforme descrito nos procedimentos do estudo foi realizada, ao final do programa, uma avaliação da satisfação e do cumprimento dos objetivos do programa na visão dos tutorandos. Os principais pontos positivos levantados pelos tutorandos em relação ao programa de tutoria por pares foram: esclarecimento de dúvidas em relação à faculdade e ao curso $(f=6)$; troca de experiências entre tutor e tutorando $(f=5)$; auxílio na gestão do tempo $(f=4)$; efetividade das atividades práticas propostas $(f=3)$; diversidade de temas abordados $(f=2)$; e estrutura dos materiais $(f=2)$.

Dois dos tutorandos ressaltaram terem percebido mudanças positivas em sua rotina, que acreditam terem sido facilitadas pelos conteúdos abordados na tutoria. Como exemplo, um dos tutorandos comentou que iniciou uma atividade em uma entidade da faculdade e começou a buscar informações para iniciar uma iniciação científica, sendo esses dois tópicos tratados no Módulo 1 Vida Universitária.

Dois tutorandos consideraram que o programa os auxiliou por meio do relacionamento construído com o tutor. De acordo com eles, ter tido a chance de conversar com um veterano de seu curso foi importante, já que havia uma abertura maior para tirar suas dúvidas.

\section{Avaliação da disciplina-estágio e do Programa de Tutoria por Pares pelos Tutores}

Foi realizada a avaliação da disciplina-estágio pelos 9 tutores, conforme Tabela 5. Verificou-se que na maioria dos itens obtiveram-se notas acima da média, o que indica que os alunos avaliaram positivamente a disciplina e o aprendizado. 
Tabela 5

Avaliação geral dos tutores em relação à disciplina-estágio

\begin{tabular}{lccccc}
\hline \multicolumn{1}{c}{ Itens avaliados } & \multirow{2}{*}{ M } & DP & \multicolumn{2}{c}{ Concentração de respostas } \\
\cline { 5 - 6 } & & & $1-2$ & 3 & $4-5$ \\
\hline 1. Pertinência do conteúdo & 4.12 & 0.35 & 0 & 0 & 100 \\
2. Estratégias de ensino utilizadas no desenvolvimento do conteúdo & 4.0 & 0.53 & 0 & 12.5 & 87.5 \\
3. Cumprimento dos objetivos propostos & 3.8 & 0.35 & 0 & 12.5 & 87.5 \\
4. Participação em sala de aula & 4.5 & 0.53 & 0 & 0 & 100 \\
5. Participação geral dos discentes durante as atividades & 4.5 & 0.53 & 0 & 0 & 100 \\
6. Atuação docente durante as aulas e atividades & 4.5 & 0.53 & 0 & 0 & 100 \\
7. Critérios de avaliação & 4.8 & 0.35 & 0 & 0 & 100 \\
8. Atividades de avaliação propostas & 4.8 & 0.35 & 0 & 0 & 100 \\
9. Materiais de apoio ao aprendizado & 3.8 & 0.64 & 0 & 25 & 75 \\
10. Bibliografia & 4 & 0.53 & 0 & 12.5 & 87.5 \\
11. Relação entre tempo dispendido e aprendizado conseguido & 4 & 0.75 & 0 & 25 & 75 \\
12. Relação entre docentes e alunos & 4.8 & 0.35 & 0 & 0 & 100 \\
13. Relação entre alunos e alunos & 4.7 & 0.4 & 0 & 0 & 100 \\
\hline
\end{tabular}

Em relação às questões abertas, os alunos destacaram como pontos positivos da disciplina: contato com a prática de atuação, dando-lhes maior autonomia na execução de seu papel como tutor; a montagem dos materiais lhes garantiu melhor absorção de conhecimento; as estratégias de ensino e o material de apoio utilizado, em conjunto com o formato interativo das aulas foram importantes para o bom aprendizado; a colaboração das docentes e dos alunos foi um aspecto muito importante na qualidade da disciplina.

Em relação aos aspectos destacados que poderiam ser aperfeiçoados têm-se: a organização do cronograma, principalmente no que diz respeito ao início do programa de tutoria, que deveria ser antecipado para evitar o final do semestre; maior aprofundamento no conteúdo dos módulos; e revisão da bibliografia utilizada.

Quanto ao programa de Tutoria por Pares, foram citados como pontos positivos para o próprio tutor: desenvolvimento pessoal $(f=2)$; satisfação pessoal $(f=6)$; desenvolvimento de habilidades de escuta $(f=3)$; contato com a prática $(f=8)$.

\section{Considerações finais}

De acordo com os resultados encontrados, observou-se que os estudantes universitários que finalizaram o programa de tutoria por pares avaliaram positivamente $\mathrm{o}$ programa e relataram que suas demandas iniciais foram atendidas, como falta de informação em relação à universidade e ao curso, gestão do tempo e troca de experiências. Outro conjunto de dados a ser destacado foram os benefícios do programa para os estagiários/tutores, considerando o estudo dos temas trabalhados, bem como o desenvolvimento de competências sociais (Garitano, Ruiz \& Andonegui, 2017) e práticas profissionais importantes ao futuro psicólogo, em especial, habilidades de escuta, empatia, automonitoramento, autogerenciamento de necessidades de aprendizagem, e busca de aprofundamento teórico a partir das demandas apresentadas pelos tutorandos.

Em relação às habilidades sociais, os resultados encontrados neste estudo estão de acordo com os evidenciados em outras pesquisas, as quais apontam como uma demanda dos universitários em processo de adaptação a necessidade de desenvolvimento de habilidades sociais, propondo os programas de tutoria por pares como uma indicação de intervenção para os alunos em relação ao desenvolvimento de habilidades sociais (Muñoz, Barbero e Feliu, 2015). Para mensurar tal efeito, sugere-se que nas próximas edições do programa o Inventário de Habilidades Sociais seja aplicado no inicio e término do programa de tutoria por pares.

Além disso, sugere-se também que o delineamento metodológico seja alterado, de forma a incluir um grupo controle, acompanhamento do desempenho acadêmico ao longo do semestre, e inclusão de critérios objetivos, por exemplo, as notas obtidas, para mensurar o efeito do programa. Tais alterações visam superar uma dificuldade apontada em estudos anteriores (Stigmar, 2016) de se mensurar os efeitos de programas de tutoria por pares, devido às diferentes variáveis que podem interferir no contexto complexo de ensino e aprendizagem, dificultando a identificação de relações causais. 
No Brasil, a produção de estudos voltados para a avaliação da eficácia de programas de tutoria por pares é escassa. De um modo geral, observou-se que o objetivo inicial deste artigo, apresentar e analisar uma proposta de programa de tutoria por pares com foco na adaptação da população universitária, foi cumprido. Para complementar os resultados encontrados neste estudo é fundamental que sejam desenvolvidas mais pesquisas sobre o tema utilizando outras metodologias, com amostras mais robustas. Isso permitirá uma maior compreensão do processo de adaptação à vida universitária, bem como a importância do desenvolvimento de habilidades sociais neste processo.

\section{Referências}

Almeida, L. S., Ferreira, J. A. G., \& Soares, A. P. (1999). Questionário de Vivências Académicas: Construção e validação de uma versão reduzida (QVA-r). Revista Portuguesa de Pedagogia, 3, 181-207.

Almeida, L. S., Soares, A. P., \& Ferreira, J. A. G. (2002). Questionário de Vivências Acadêmicas (QVA-r): Avaliação do ajustamento dos estudantes universitários. Avaliação Psicológica, 1(2), 81-93.

Alpay, E., Cutler, P. S., Eisenbach, S., \& Field, A. J. (2010). Changing the marks-based culture of learning through peerassisted tutorials. European Journal of Engineering Education, 35(1), 17-32.

Bandeira, M., Neves, M. C., Del Prette, Z. A. P., Del Prette, A., \& Gerk, E. C. (2000). Qualidades psicométricas do Inventário de Habilidades Sociais (IHS): estudo sobre a estabilidade temporal e a validade concomitante. Estudos de psicologia, 5(2), 401-419.

Bowling, B., Doyle, M., Taylor, J., \& Antes, A. (2015). Professionalizing the role of peer leaders in STEM. Journal of STEM Education: Innovations and Research, 16(2), 30-39.

Cardozo-Ortiz, C. E. (2011). Tutoria entre pares como uma estratégia pedagógica univeristaria. Educácion y Educadores 14(2), 309-325.

Collazo, M., Hernández, O., \& Seoane, M. (2014). La tutoría entre pares: primera experiencia curricular opcional en la Facultad de Odontología-UDELAR. Odontoestomatología, 16(23), 54-66.

Cunha, S. M., \& Carrilho, D. M. (2005). O processo de adaptação ao ensino superior e o rendimento acadêmico. Psicologia escolar e educacional, 9(2), 215-224.

De Smet, M., Van Keer, H., \& Valcke, M. (2009). Cross-age peer tutors in asynchronous discussion groups: A study of the evolution in tutor support. Instructional Science, 37(1), 87-105.

Del Prette, Z. A. P., \& Del Prette, A. (2001). Habilidades sociais e educação: Pesquisa e atuação em psicologia escolar/educacional. Em Del Prette, Z. A. P. (Org.), Psicologia escolar e educacional: Saúde e qualidade de vida (pp. 113-141). Campinas, SP: Alínea.

Diniz, A. M., \& Almeida, S. L. (2006). Adaptação à universidade em estudantes do primeiro ano: Estudo diacrónico da interacção entre o relacionamento com pares, o bem-estar pessoal e o equilíbrio emocional. Análise Psicológica, XXIV(1), 29-38.

Eby, L. T., Rhodes, J. E., \& Allen, T. D. (2007). Definition and evolution of mentoring. In Allen, T. D., \& Eby, L. T. (orgs.) The Blackwell handbook of mentoring: A multiple perspectives approach. Oxford, England: Blackwell Publishing.

Faria, C., Coelho, C., Oliveira, F., Lucas, C. V., \& Soares, L. Entre Pares: Um programa de tutoria na Universidade da Madeira. Livro de Atas, 67-75.

Garitano, E. T., Ruiz, U. G., \& Andonegui, A. R. (2017). Influencia del mentoring en el rendimiento de los participantes en las comunidades virtuales de práctica. Revista Interuniversitaria de Investigación en Tecnología Educativa, (3), 40-50.

Gorlitz, K., \& Gravert, C. (2016). The effects of the high school curriculum on school dropout. Applied Economics, 48(54), 5314-5328.

Jacobi, M. (1991). Mentoring and undergraduate academic success: A literature review. Review of educational research, 61(4), 505-532.

Larose, S., Cyrenne, D., Garceau, O., Harvey, M., Guay, F., Godin, F., ... \& Deschênes, C. (2011). Academic mentoring and dropout prevention for students in math, science and technology. Mentoring \& Tutoring: Partnership in Learning, 19(4), 419-439.

Lefèvre, F., Lefèvre, A. M. C., \& Teixeira, J. J. V. (2000). O discurso do sujeito coletivo: uma nova abordagem metodológica em pesquisa qualitativa. Caxias do sul, EDUCS

Lueg, K., \& Lueg, R. (2014). From teacher-centred instruction to peer tutoring in the heterogeneous international classroom: A Danish case of instructional change. Journal of social science education, 13(2), 39-62. 
Meneses, N. F. (2018). Evasão escolar no ensino superior. Valor Econômico. Recuperado em 20 de Julho de 2018, de https://www.valor.com.br/colunistas/Naercio\%20Menezes\%20Filho

Mercuri, E. \& Polydoro, S. A. J. (Orgs.) (2003). Estudante universitário: Características e experiências de formação. Taubaté: Cabral.

Millan, L. R., Souza, E. N. D., De Marco, O. L. N., Rossi, E., \& Arruda, P. C. V. D. (1998). OI Encontro Paulista dos serviços de assistência psicológica ao estudante universitário. Rev Hosp Clin Fac Med Sao Paulo, 53(3), 156-161.

Munley, V. G., Garvey, E., \& McConnell, M. J. (2010). The effectiveness of peer tutoring on student achievement at the university level. American Economic Review, 100(2), 277-82.

Muñoz, R. C., Barbero, F. L., \& Feliu, J. C. (2015). Diez pasos clave en el desarrollo de un programa de mentoría universitaria para estudiantes de nuevo ingreso. Revista Electrónica Educare, 19(2), 155-179.

Oliveira, C. T., \& Dias, A. C. G. (2014). Dificuldades na Trajetória Universitária e Rede de Apoio de Calouros e Formandos. Psico, 45(2), 187-197.

Oliveira, R. E. C., \& Morais, A. (2015). Vivências acadêmicas e adaptação de estudantes de uma universidade pública federal do Estado do Paraná. Revista de Educação Pública, 24(57), 547-568.

Pedicchiio, M. C.; Fontana, I. (2000). Tutoring in European Universities (Tutoría en universidades europeas). Trieste: Servizio Tipogradico editoriale d'Ateneo.

Qureshi, M. A., \& Stormyhr, E. (2012). Group dynamics and peer-tutoring a pedagogical tool for learning in higher education. International Education Studies, 5(2), 118-124.

Stigmar, M. (2016). Peer-to-peer teaching in higher education: A critical literature review. Mentoring \& Tutoring: Partnership in Learning, 24(2), 124-136.

Teixeira, M. A. P., Dias, A. C. G., Wottrich, S. H., \& Oliveira, A. M. (2008). Adaptação à universidade em jovens calouros. Psicologia escolar e educacional, 12(1), 185-202.

Topping, K. J. (1996). The effectiveness of peer tutoring in further and higher education: A typology and review of the literature. Higher education, 32(3), 321-345.

Topping, K., Miller, D., Thurston, A., McGavock, K., \& Conlin, N. (2011). Peer tutoring in reading in Scotland: thinking big. Literacy, 45(1), 3-9.

Underhill, J., \& McDonald, J. (2010). Collaborative tutor development: Enabling a transformative paradigm in a South African University. Mentoring \& Tutoring: Partnership in Learning, 18(2), 91-106.

Wilson, W. L., \& Arendale, D. R. (2011). Peer educators in learning assistance programs: Best practices for new programs. New Directions for Student Services, 2011(133), 41-53.

Recebido 30/01/2018

$1^{a}$ Reformulação 19/07/2018

Aceite Final 07/10/2018

Sobre as autoras

Carolina Estevam é Graduanda em Psicologia pela Faculdade de Filosofia, Ciências e Letras de Ribeirão Preto Universidade de São Paulo (FFCLRP/USP), Ribeirão Preto, SP, Brasil.

Ana Júlia Basilio é Graduanda em Psicologia pela Faculdade de Filosofia, Ciências e Letras de Ribeirão Preto Universidade de São Paulo (FFCLRP/USP), Ribeirão Preto, SP, Brasil.

Marina Greghi Sticca é Professora Doutora em Psicologia Organizacional e do Trabalho da Faculdade de Filosofia, Ciências e Letras de Ribeirão Preto - Universidade de São Paulo (FFCLRP/USP), Ribeirão Preto, SP, Brasil. Ênfase das pesquisas em ergonomia, atuando principalmente nos seguintes temas: saúde do trabalhador, carga mental de trabalho e organização do trabalho.

Fabiana Maris Versuti é Professora do Departamento de Psicologia, na área de Fundamentos Psicológicos da Educação e Ensino de Ciências. Coordenadora do curso de Licenciatura em Ciências-Pólo de Ribeirão Preto. Docente do Programa de Pós-Graduação em Psicobiologia da Faculdade de Filosofia, Ciências e Letras de Ribeirão Preto (FFCLRP) da Universidade de São Paulo (USP). 